\title{
Impact of Pump-Probe Time Delay on the Four Wave Mixing Conversion Efficiency in Semiconductor Optical Amplifiers
}

\author{
Narottam Das ${ }^{1}$, Hitoshi Kawaguchi ${ }^{2}$ and Kamal Alameh ${ }^{1,3}$ \\ ${ }^{1}$ Electron Science Research Institute, Edith Cowan University, \\ ${ }^{2}$ Graduate School of Materials Science, Nara Institute of Science and Technology, \\ ${ }^{3}$ Department of Nanobio Materials and Electronics, \\ Gwangju Institute of Science and Technology, \\ ${ }^{1}$ Australia \\ ${ }^{2}$ Japan \\ ${ }^{3}$ Republic of Korea
}

\section{Introduction}

Four-wave mixing (FWM) in semiconductor optical amplifiers (SOAs) have attracted much attention especially for applications involving fast wavelength conversion and optical demultiplexing (Mecozzi et al., 1995; Mecozzi \& Mфrk, 1997; Das et al., 2000). The optimisation of the time delay between the input pump and probe pulses is crucial for maximising the FWM conversion efficiency and reducing the timing jitter (Inoue \& Kawaguchi, 1998b; Das et al., 2005). The FWM conversion efficiency is mainly limited by the gain saturation of the SOA, which is strongly dependent on the pulse duration, repetition rate of the input pulses, and time delay between pump and probe pulses. Therefore, it is important to analyse theoretically and investigate experimentally the dependence of the FWM conversion efficiency on the time delay between the input pump and probe pulses in SOAs. The impact of the time-delay between sub-picosecond optical pump and probe pulses on the FWM conversion efficiency in SOAs was experimentally observed for the first time and reported by Inoue and Kawaguchi (Inoue \& Kawaguchi, 1998b). A preliminary theoretical analysis for evaluating the FWM conversion efficiency of SOAs was reported by Das et al. (Das et al., 2005).

The optimization of the time delay between the optical pump and probe pulses is very important in order to achieve a high FWM conversion efficiency and minimize the timing jitter (Inoue \& Kawaguchi, 1998b; Das et al., 2005). Shtaif et al., (Shtaif \& Eisenstein, 1995; Shtaif et al., 1995) have investigated analytically and experimentally the dependence of the FWM conversion efficiency for short optical pulses in SOA. They measured the FWM conversion efficiency for short optical pulses in the order of $10 \mathrm{ps}$ and suggested that the high FWM conversion efficiency can be obtained when short optical pulses are used. The FWM characteristics for subpicosecond time-delays between the input optical pulses in SOAs have been reported. However, no theoretical analysis (Inoue \& Kawaguchi, 1998b) was reported. Therefore, it is very important to analyze theoretically and measure 
experimentally the FWM conversion efficiency in SOAs for subpicosecond optical pulses and optimize the time-delay between the input pump and probe pulses.

In this Chapter, we present a comprehensive analysis based on the finite-difference beam propagation method (FD-BPM) for evaluating the FWM conversion efficiency in SOAs and discuss the experimental results reported on the optimisation of the time delay between pump and probe pulses to maximize the FWM conversion efficiency. This comprehensive analysis is crucial not only for attaining high FWM conversion efficiency but also for characterizing the timing jitter. Simulation results demonstrate that, for short optical pulses, the time delay between the pump and probe pulses at the input of the SOA can be optimized to maximize the FWM conversion efficiency. Excellent agreement between the simulation and experimental results is achieved, demonstrating a high FWM conversion efficiency in SOAs when the time delay between the input pump and probe pulses is optimized.

The FD-BPM can obtain the FWM characteristics for different propagation scenarios using a modified nonlinear Schrödinger equation (MNLSE) (Hong et al., 1996 \& Das et al., 2000), simply by changing only the combination of input optical pulses. These scenarios are: (1) single pulse propagation (Das et al., 2008), (2) two input pulses (Das et al., 2000), (3) multiplexing of several input pulses (Das et al., 2001), (4) two input pulses with phaseconjugation (Das et al., 2001), and (5) two input pulses with optimum time-delay between them (Das et al., 2007).

The analyses are based on the MNLSE considering the group velocity dispersion (GVD), self-phase modulation, and two-photon absorption, with the dependencies on the carrier depletion, carrier heating, spectral-hole burning and their dispersions, including the recovery times in SOAs (Hong et al., 1996). The simulation results show that the FWM conversion efficiency increases with the optimum time-delay between the input pump and probe pulses and are in excellent agreement with the experiment results.

\section{Theoretical model}

In this section, we briefly discuss the nonlinear effects in SOAs, the formulation of modified nonlinear Schrödinger equation (MNLSE), and the finite-difference beam propagation method (FD-BPM) used in the simulation, the nonlinear propagation characteristics of solitary pulses, the FWM characteristics in SOAs, and the FWM characteristics for various time-delays between the input pump and pulses.

\subsection{Nonlinear effects in SOAs}

There are different types of "nonlinear effects" in SOAs. Among them, four types of important optical "nonlinear effects" are explained. These are (i) spectral hole-burning (SHB), (ii) carrier heating $(\mathrm{CH})$, (iii) carrier depletion (CD), and (iv) two-photon absorption (TPA).

Figure 1 shows the time-development of the population density in the conduction band after excitation. The arrow (pump) shown in Fig. 1 is the excitation laser energy. Below $100 \mathrm{fs}$, the SHB effect is dominant. SHB occurs when a narrow-band strong pump beam excites the SOA, which has an inhomogeneous broadening. SHB arises due to the finite value of intraband carrier-carrier scattering time $(\sim 50-100 \mathrm{fs})$, which sets the time scale on which a quasi-equilibrium Fermi distribution is established among the carriers in a band. After about 1 ps, the SHB effect is relaxed and the $\mathrm{CH}$ effect becomes dominant. The process tends 

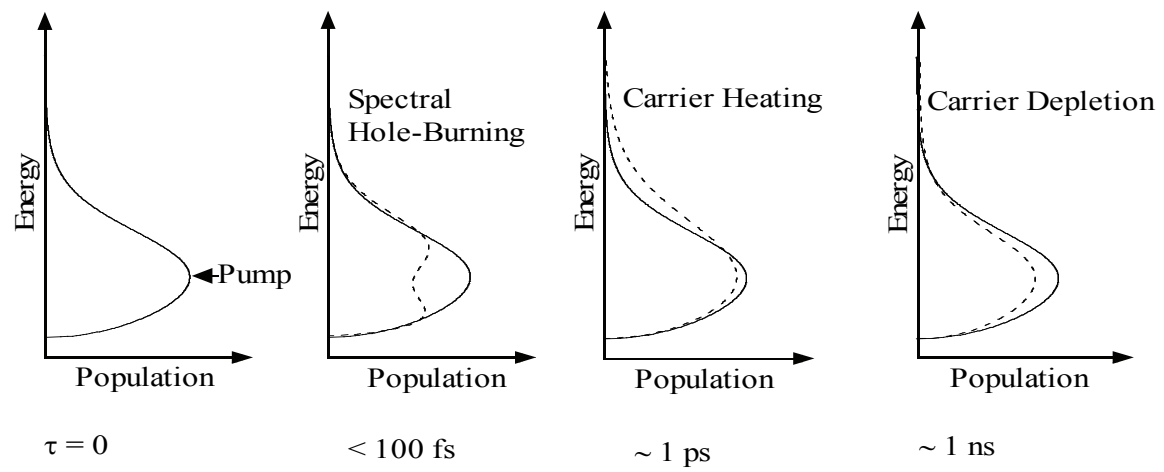

Fig. 1. Nonlinear effects in SOAs. The main effects are: (i) spectral hole-burning (SHB) with a life time of $<100 \mathrm{fs}$, (ii) carrier heating $(\mathrm{CH})$ with a life time of $\sim 1 \mathrm{ps}$, (iii) carrier depletion (CD) with a life time is $\sim 1 \mathrm{~ns}$, and (iv) two-photon absorption (TPA).

to increase the temperature of the carriers beyond the lattice's temperature. The main causes of heating the carriers are (1) the stimulated emission, since it involves the removal of "cold" carriers close to the band edge, and (2) the free-carrier absorption, which transfers carriers to high energies within the bands. The "hot"-carriers relax to the lattice temperature through the emission of optical phonons with a relaxation time of $\sim 0.5-1 \mathrm{ps}$. The effect of carrier depletion (CD) remains for about $1 \mathrm{~ns}$. The stimulated electron-hole recombination depletes the carriers, thus reducing the optical gain. The band to band relaxation also causes carrier depletion, with a relaxation time $\sim 0.2-1$ ns. For ultrashort optical pumping, the twophoton absorption (TPA) effect also becomes important. An atom makes a transition from its ground state to the excited state by the simultaneous absorption of two laser photons. All these mechanisms (effects) are taken into account in the simulation and the formulation of modified nonlinear Schrödinger equation (MNLSE).

\subsection{Formulation of Modified Nonlinear Schrödinger Equation (MNLSE)}

In this subsection, we will briefly explain the theoretical analysis of short optical pulses propagation in SOAs. Starting from Maxwell's equations (Agrawal, 1989; Yariv, 1991; Sauter, 1996), we reach the propagation equation of short optical pulses in SOAs which are governed by the wave equation (Agrawal \& Olsson, 1989) in the frequency domain:

$$
\nabla^{2} \bar{E}(x, y, z, \omega)+\frac{\varepsilon_{r}}{c^{2}} \omega^{2} \bar{E}(x, y, z, \omega)=0
$$

where, $\bar{E}(x, y, z, \omega)$ is the electromagnetic field of the pulse in the frequency domain, $\mathrm{c}$ is the velocity of light in vacuum and $\varepsilon_{r}$ is the nonlinear dielectric constant which is dependent on the electric field in a complex form. By slowly varying the envelope approximation and integrating the transverse dimensions we arrive at the pulse propagation equation in SOAs (Agrawal \& Olsson, 1989; Dienes et al., 1996).

$$
\frac{\partial V(\omega, z)}{\partial z}=-i\left\{\frac{\omega}{c}\left[1+\chi_{m}(\omega)+\Gamma \tilde{\chi}(\omega, N)\right]^{1 / 2}-\beta_{0}\right\} V(\omega, z)
$$


where, $V(\omega, z)$ is the Fourier-transform of $V(t, z)$ representing pulse envelope, $\chi_{m}(\omega)$ is the background (mode and material) susceptibility, $\tilde{\chi}(\omega)$ is the complex susceptibility which represents the contribution of the active medium, $N$ is the effective population density, $\beta_{0}$ is the propagation constant. The quantity $\Gamma$ represents the overlap/ confinement factor of the transverse field distribution of the signal with the active region as defined in (Agrawal \& Olsson, 1989).

Using mathematical manipulations (Sauter, 1996; Dienes et al., 1996), including the real part of the instantaneous nonlinear Kerr effect as a single nonlinear index $\mathrm{n}_{2}$ and by adding the two-photon absorption (TPA) term we obtain the MNLSE for the phenomenological model of semiconductor laser and amplifiers (Hong et al., 1996). The following MNLSE (Hong et al., 1996; Das et al., 2000) is used for the simulation of FWM characteristics with optimum time-delays between pulses in SOAs:

$$
\begin{aligned}
& {\left[\frac{\partial}{\partial z}-\frac{i}{2} \beta_{2} \frac{\partial^{2}}{\partial \tau^{2}}+\frac{\gamma}{2}+\left(\frac{\gamma_{2 p}}{2}+i b_{2}\right)|V(\tau, z)|^{2}\right] V(\tau, z)} \\
& \quad=\left\{\frac{1}{2} g_{N}(\tau)\left[\frac{1}{f(\tau)}+i \alpha_{N}\right]+\frac{1}{2} \Delta g_{T}(\tau)\left(1+i \alpha_{T}\right)-\left.i \frac{1}{2} \frac{\partial g(\tau, \omega)}{\partial \omega}\right|_{\omega_{0}} \frac{\partial}{\partial \tau}-\left.\frac{1}{4} \frac{\partial^{2} g(\tau, \omega)}{\partial \omega^{2}}\right|_{\omega_{0}} \frac{\partial^{2}}{\partial \tau^{2}}\right\} V(\tau, z)
\end{aligned}
$$

We introduce the frame of local time $\tau\left(=t-\mathrm{z} / \mathrm{v}_{g}\right)$, which propagates with a group velocity $\mathrm{v}_{g}$ at the center frequency of an optical pulse. A slowly varying envelope approximation is used in (3), where the temporal variation of the complex envelope function is very slow compared with the cycle of the optical field. In (3), $V(\tau, z)$ is the time domain complex envelope function of an optical pulse, $|V(\tau, z)|^{2}$ corresponding to the optical power, and $\beta_{2}$ is the GVD. $\gamma$ is the linear loss, $\gamma_{2 p}$ is the two-photon absorption coefficient, $b_{2}\left(=\omega_{0} n_{2} / c A\right)$ is the instantaneous self-phase modulation term due to the instantaneous nonlinear Kerr effect $n_{2}, \omega_{0}\left(=2 \pi f_{0}\right)$ is the center angular frequency of the pulse, $c$ is the velocity of light in vacuum, $A(=w d / \Gamma)$ is the effective area $(d$ and $w$ are the thickness and width of the active region, respectively, and $\Gamma$ is the confinement factor) of the active region.

The saturation of the gain due to the carrier depletion is given by (Hong et al., 1996)

$$
g_{N}(\tau)=g_{0} \exp \left(-\frac{1}{W_{s}} \int_{-\infty}^{\tau} e^{-s / \tau_{s}}|V(s)|^{2} d s\right)
$$

where, $g_{N}(\tau)$ is the saturated gain due to carrier depletion, $g_{0}$ is the linear gain, $W_{s}$ is the saturation energy, $\tau_{s}$ is the carrier lifetime.

The spectral hole-burning (SHB) function $f(\tau)$ is given by (Hong et al., 1996)

$$
f(\tau)=1+\frac{1}{\tau_{\text {shb }} P_{s h b}} \int_{-\infty}^{+\infty} u(s) e^{-s / \tau_{s h b}}|V(\tau-s)|^{2} d s
$$

where, $f(\tau)$ is the SHB function, $P_{\text {shb }}$ is the spectral hole-burning saturation power, $\tau_{\text {shb }}$ is the spectral hole-burning relaxation time, and $\alpha_{N}$ and $\alpha_{T}$ are the linewidth enhancement factor associated with the gain changes due to the carrier depletion and carrier heating. The resulting gain change due to the $\mathrm{CH}$ and TPA is given by (Hong et al., 1996) 


$$
\begin{aligned}
\Delta g_{T}(\tau) & =-h_{1} \int_{-\infty}^{+\infty} u(s) e^{-s / \tau_{c h}}\left(1-e^{-s / \tau_{s l b}}\right)|V(\tau-s)|^{2} d s \\
& -h_{2} \int_{-\infty}^{+\infty} u(s) e^{-s / \tau_{c h}}\left(1-e^{-s / \tau_{s l b}}\right)|V(\tau-s)|^{4} d s
\end{aligned}
$$

where, $\Delta g_{T}(\tau)$ is the resulting gain change due to the $\mathrm{CH}$ and TPA, $u(\mathrm{~s})$ is the unit step function, $\tau_{c h}$ is the carrier heating relaxation time, $h_{1}$ is the contribution of stimulated emission and free-carrier absorption to the carrier heating gain reduction and $h_{2}$ is the contribution of two-photon absorption.

The dynamically varying slope and curvature of the gain plays a shaping role for pulses in the sub-picosecond range. The first and second order differential net (saturated) gain terms are (Hong et al., 1996),

$$
\begin{gathered}
\left.\frac{\partial g(\tau, \omega)}{\partial \omega}\right|_{\omega_{0}}=A_{1}+B_{1}\left[g_{0}-g\left(\tau, \omega_{0}\right)\right] \\
\left.\frac{\partial^{2} g(\tau, \omega)}{\partial \omega^{2}}\right|_{\omega_{0}}=A_{2}+B_{2}\left[g_{0}-g\left(\tau, \omega_{0}\right)\right] \\
g\left(\tau, \omega_{0}\right)=g_{N}\left(\tau, \omega_{0}\right) / f(\tau)+\Delta g_{T}\left(\tau, \omega_{0}\right)
\end{gathered}
$$

where, $A_{1}$ and $A_{2}$ are the slope and curvature of the linear gain at $\omega_{0}$, respectively, while $B_{1}$ and $B_{2}$ are constants describing changes in $A_{1}$ and $A_{2}$ with saturation, as given in (7) and (8). The gain spectrum of an SOA is approximated by the following second-order Taylor expansion in $\Delta \omega$ :

$$
g(\tau, \omega)=g\left(\tau, \omega_{0}\right)+\left.\Delta \omega \frac{\partial g(\tau, \omega)}{\partial \omega}\right|_{\omega_{0}}+\left.\frac{(\Delta \omega)^{2}}{2} \frac{\partial^{2} g(\tau, \omega)}{\partial \omega^{2}}\right|_{\omega_{0}}
$$

The coefficients $\left.\frac{\partial g(\tau, \omega)}{\partial \omega}\right|_{\omega_{0}}$ and $\left.\frac{\partial^{2} g(\tau, \omega)}{\partial \omega^{2}}\right|_{\omega_{0}}$ are related to $A_{1}, B_{1}, A_{2}$ and $B_{2}$ by (7) and (8). Here we assumed the same values of $A_{1}, B_{1}, A_{2}$ and $B_{2}$ as in (Hong et al., 1996) for an AlGaAs/GaAs bulk SOA.

The time derivative terms in (3) have been replaced by the central-difference approximation in order to simulate this equation by the FD-BPM (Das et al., 2000). In simulation, the parameter of bulk SOAs (AlGaAs/GaAs, double heterostructure) with a wavelength of 0.86 $\mu \mathrm{m}$ (Hong et al., 1996) is used and the SOA length is $350 \mu \mathrm{m}$. The input pulse shape is sech ${ }^{2}$ and is Fourier transform-limited.

The gain spectra of SOAs are important for obtaining the propagation and wave mixing (FWM and FWM with time-delay between the input pump and probe pulses) characteristics of short optical pulses. Figure 2 shows the gain spectra given by a second-order Taylor expansion about the pulse center frequency with derivatives of $g(\tau, \omega)$ by $(7)$ and (8). The solid line shows the unsaturated gain spectrum having $\mathrm{g}_{0}(=g(\tau, \omega))$ of $92 \mathrm{~cm}^{-1}$ at $\omega_{0}$. The dotted line is a saturated gain spectrum having $g_{0} / 2\left(=46 \mathrm{~cm}^{-1}\right)$ at $\omega_{0}$, and the dashed-dotted line is a strongly saturated gain spectrum having $g(\tau, \omega)$ of $0 \mathrm{~cm}^{-1}$ at $\omega_{0}$. The pump frequency $\omega_{0}$ is set to near the gain peak, and the linear gain $\mathrm{g}_{0}$ is $92 \mathrm{~cm}^{-1}$ at $\omega_{0}$. 


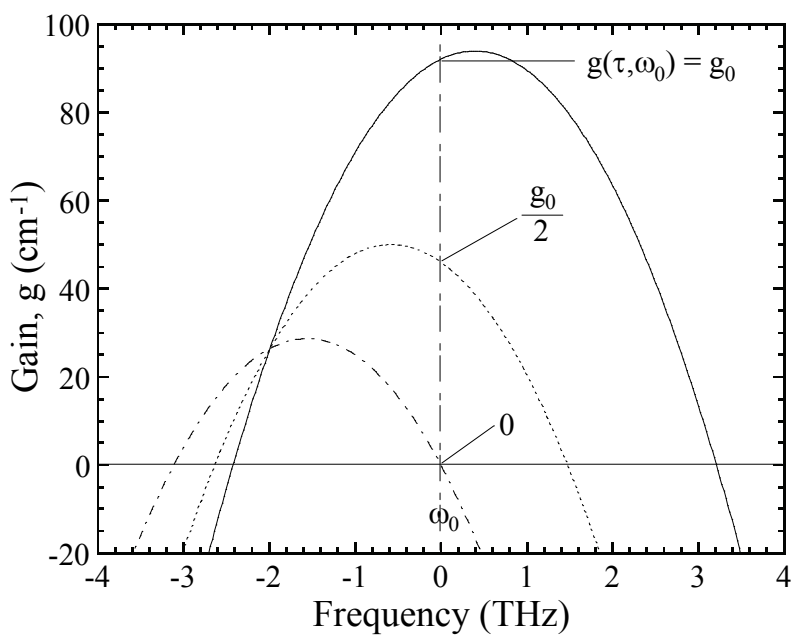

Fig. 2. The gain spectra given by the second-order Taylor expansion about the pulse center frequency, with the derivatives of $g(\tau, \omega)$ by $(7)$ and (8). The upper curve is linear gain, $\mathrm{g}_{0}=92 \mathrm{~cm}^{-1}$, middle curve is half-saturated gain, $\mathrm{g}_{0} / 2=46 \mathrm{~cm}^{-1}$, and lower curve is fully saturated gain, $\mathrm{g}_{0}=0$.

The gain bandwidth is about the same as the measured value for an AlGaAs/GaAs bulk SOA (Seki et al., 1981). If an InGaAsP/InP bulk SOA is used we can expect much wider gain bandwidth (Leuthold et al., 2000). With a decrease in the carrier density, the gain decreases and the peak position is shifted to a lower frequency because of the band-filling effect.

This MNLSE was initially used by (Hong et al., 1996) for the analysis of a "solitary pulse" propagation in an SOA. We used the same MNLSE for the simulation of FWM in an SOA using the FD-BPM. We have introduced a complex envelope function $\mathrm{V}(\tau, 0)$ at the input side of the SOA for taking into account the two (pump and probe) pulses.

\subsection{Finite-Difference Beam Propagation Method (FD-BPM)}

To solve a boundary value problem by the finite-differences method, every derivative appearing in the equation, as well as in the boundary conditions, is replaced by the central differences approximation. Central differences are usually preferred because they lead to greater accuracy (Conte \& Boor, 1980). In our simulations, we used the finite-differences (central differences) to solve the MNLSE for this research work.

Usually, the fast Fourier transformation beam propagation method (FFT-BPM) (Okamoto, 1992; Brigham, 1988) is used for the analysis of the optical pulse propagation in optical fibers by the successive iterations of the Fourier transformation and the inverse Fourier transformation. In the FFT-BPM, the linear propagation term (GVD term) and phase compensation terms (other than GVD, 1st and 2nd order gain spectrum terms) are separated in the nonlinear Schrödinger equation for the individual consideration of the time and frequency domain for the optical pulse propagation. However, in our model, equation (3) includes the dynamic gain change terms, i.e., the 1st and 2nd order gain spectrum terms which are the last two terms of the right-side in equation (3). Therefore, it is not possible to 
separate equation (3) into the linear propagation term and phase compensation term and it is quite difficult to calculate equation (3) using the FFT-BPM. For this reason, we used the FD-BPM (Chung \& Dagli, 1990; Conte \& Boor, 1980). If we replace the time derivative terms of equation (3) by the below central-difference approximation, equation (11), and integrate equation (3) with the small propagation step $\Delta \mathrm{z}$, we obtain the tridiagonal simultaneous matrix equation (12)

$$
\frac{\partial}{\partial \tau} V_{k}=\frac{V_{k+1}-V_{k-1}}{2 \Delta \tau}, \frac{\partial^{2}}{\partial \tau^{2}} V_{k}=\frac{V_{k+1}-2 V_{k}+V_{k-1}}{\Delta \tau^{2}}
$$

where, $V_{k}=V\left(\tau_{k}\right), V_{k+1}=V\left(\tau_{k}+\Delta \tau\right)$, and $V_{k-1}=V\left(\tau_{k}-\Delta \tau\right)$

$$
\begin{gathered}
-a_{k}(z+\Delta z) V_{k-1}(z+\Delta z)+\left\{1-b_{k}(z+\Delta z)\right\} V_{k}(z+\Delta z)-c_{k}(z+\Delta z) V_{k+1}(z+\Delta z) \\
=a_{k}(z) V_{k-1}(z)+\left\{1+b_{k}(z)\right\} V_{k}(z)+c_{k}(z) V_{k+1}(z)
\end{gathered}
$$

where, $k=1,2,3$, $n$ and

$$
\begin{aligned}
a_{k}(z)= & \frac{\Delta z}{2}\left[\frac{i \beta_{2}}{2 \Delta \tau^{2}}+\left.i \frac{1}{4 \Delta \tau} \frac{\partial g(\tau, \omega, z)}{\partial \omega}\right|_{\omega_{0}, \tau_{k}}-\left.\frac{1}{4 \Delta \tau^{2}} \frac{\partial^{2} g(\tau, \omega, z)}{\partial \omega^{2}}\right|_{\omega_{0}, \tau_{k}}\right] \\
b_{k}(z)= & -\frac{\Delta z}{2}\left[\frac{i \beta_{2}}{\Delta \tau^{2}}+\frac{\gamma}{2}+\left(\frac{\gamma_{2 p}}{2}+i b_{2}\right)\left|V_{k}(z)\right|^{2}-\frac{1}{2} g_{N}\left(\tau_{k}, \omega_{0}, z\right)\left(1+i \alpha_{N}\right)\right. \\
& \left.-\frac{1}{2} \Delta g_{T}\left(\tau_{k}, \omega_{0}, z\right)\left(1+i \alpha_{T}\right)-\left.\frac{1}{2 \Delta \tau^{2}} \frac{\partial^{2} g(\tau, \omega, z)}{\partial \omega^{2}}\right|_{\omega_{0}, \tau_{k}}\right] \\
c_{k}(z)= & \frac{\Delta z}{2}\left[\frac{i \beta_{2}}{2 \Delta \tau^{2}}-\left.i \frac{1}{4 \Delta \tau} \frac{\partial g(\tau, \omega, z)}{\partial \omega}\right|_{\omega_{0}, \tau_{k}}-\left.\frac{1}{4 \Delta \tau^{2}} \frac{\partial^{2} g(\tau, \omega, z)}{\partial \omega^{2}}\right|_{\omega_{0}, \tau_{k}}\right]
\end{aligned}
$$

where, $\Delta \tau$ is the sampling time and $n$ is the number of sampling. If we know $V_{k}(z)$, $(k=1,2,3, \ldots \ldots \ldots, n)$ at the position $z$, we can calculate $V_{k}(z+\Delta z)$ at the position of $z+\Delta z$ which is the propagation of a step $\Delta z$ from position $z$, by using equation (12). It is not possible to directly calculate equation (12) because it is necessary to calculate the left-side terms $a_{k}(z+\Delta z), b_{k}(z+\Delta z)$, and $c_{k}(z+\Delta z)$ of equation (12) from the unknown $V_{k}(z+\Delta z)$. Therefore, we initially defined $a_{k}(z+\Delta z) \equiv a_{k}(z), b_{k}(z+\Delta z) \equiv b_{k}(z)$, and $c_{k}(z+\Delta z) \equiv c_{k}(z)$ and obtained $V_{k}^{(0)}(z+\Delta z)$, as the zeroth order approximation of $V_{k}(z+\Delta z)$ by using equation (12). We then substituted $V_{k}^{(0)}(z+\Delta z)$ in equation (12) and obtained $V_{k}^{(1)}(z+\Delta z)$ as the first order approximation of $V_{k}(z+\Delta z)$ and finally obtained the accurate simulation results by the iteration as used in (Brigham, 1988; Chung \& Dagli, 1990).

Figure 3 shows the schematic diagram of the FD-BPM in time domain scale. Here, $\tau\left(=t-z / v_{g}\right)$ is the local time, which propagates with the group velocity $v_{g}$ at the center frequency of an optical pulse and $\Delta \tau$ is the sampling time. $\mathrm{z}$ is the propagation direction and $\Delta \mathrm{z}$ is the propagation step. With this procedure, we used up to 3-rd time iteration for more accuracy of the simulations. 


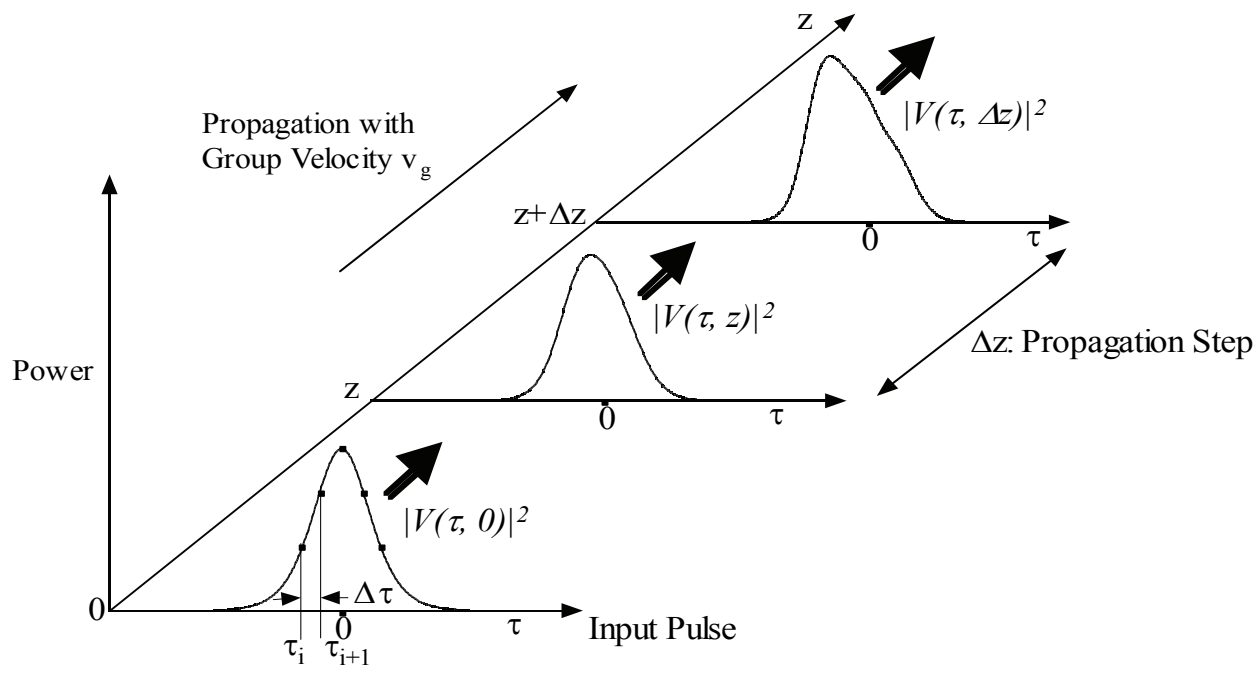

Sampling Time: $\Delta \tau$

Fig. 3. Schematic diagram of FD-BPM in time domain. $\tau\left(=t-z / v_{g}\right)$ is the local time, which propagates with the group velocity $v_{g}$ at the center frequency of an optical pulse and $\Delta \tau$ is the sampling time, and $z$ is the propagation direction and $\Delta z$ is the propagation step.

The FD-BPM (Conte \& Boor, 1980; Chung \& Dagli, 1990) is used for the simulation of several important charactreristics, namely, (1) single pulse propagation in SOAs (Das et al., 2008; Razaghi et al., 2009a \& 2009b), (2) two input pulses propagating in SOAs (Das et al., 2000; ; Connelly et al., 2008), (3) multiplexing of several input pulses using FWM (Das et al., 2001), (4) two input pulses with phase-conjugation propagating along SOAs (Das et al., 2001), and (5) two propagating input pulses with time-delay between them being optimized (Das et al., 2007).

\subsection{Optical pulse propagation in SOAs}

Optical pulse propagation in SOAs has drawn considerable attention due to its potential applications in optical communication systems, such as a wavelength converter based on FWM and switching. The advantages of using SOAs include the amplification of small (weak) optical pulses and the realization of high efficient FWM.

We analyzed the optical pulse propagation in SOAs using the FD-BPM in conjunction with the MNLSE, where several parameters are taken into account, namely, the group velocity dispersion, self-phase modulation (SPM), and two-photon absorption (TPA), as well as the dependencies on the carrier depletion, carrier heating $(\mathrm{CH})$, spectral-hole burning $(\mathrm{SHB})$ and their dispersions, including the recovery times in an SOA (Hong et al., 1996). We also considered the gain spectrum (as shown in Fig. 1). The gain in an SOA was dynamically changed depending on values used for the carrier density and carrier temperature in the propagation equation (i.e., MNLSE).

Initially, (Hong et al., 1996) used the MNLSE for the simulation of optical pulse propagation in an SOA by FFT-BPM (Okamoto, 1992; Brigham, 1988) but the dynamic gain terms were 
changing with time. The FD-BPM enables the simulation of optical pulse propagation taking into consideration the dynamic gain terms in SOAs (Das et al., 2007; Razaghi et al., 2009a \& 2009b; Aghajanpour et al., 2009). We used the modified MNLSE for optical pulse propagation in SOAs by the FD-BPM (Chung \& Dagli, 1990; Conte \& Boor, 1980). We used the FD-BPM for the simulation of FWM characteristics when input pump and probe pulses, which are delayed with respect to one another, propagate in SOAs.

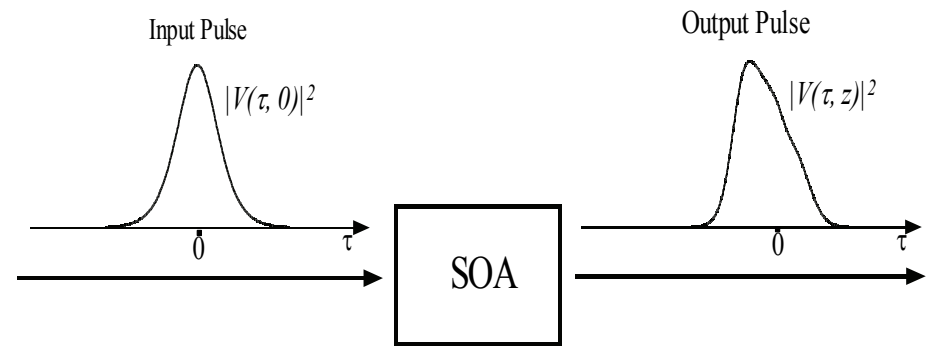

Fig. 4. Schematic diagram for the simulation of nonlinear propagation of single pulse's in SOA. Here, $|V(\tau, 0)|^{2}$ and $|V(\tau, z)|^{2}$ are the intensity of input $(\mathrm{z}=0)$ and output (after propagating a distance $z)$ pulses of SOA.

Figure 4 illustrates the simulation model for nonlinear propagation characteristics of a single pulse in an SOA. An optical pulse is injected into the input side of the SOA $(z=0)$. Here, $\tau$ is the local time, $|V(\tau, 0)|^{2}$ is the intensity (power) of input pulse at the input side of SOA ( $\mathrm{z}$ $=0$ ) and $|V(\tau, z)|^{2}$ is the intensity (power) of the output pulse at the output side of SOA after propagating a distance $\mathbf{z}$. We also used this model to simulate FWM characteristics of SOAs for multi-pulse propagation.

Figure 5 shows the simulation results for single optical pulse propagation in an SOA. Figure 5(a) shows the temporal response of the propagated pulse for different output energy levels. In this simulation, the SOA length was $500 \mu \mathrm{m}$ (other parameters are listed in Table 1) and the input pulse width was 1 ps. By increasing the input pulse energy, the output pulse energy increased until it saturated the gain of the SOA. The shift in pulse peak positions towards the leading edge (negative time) is mainly due to the gain saturation of the SOA (Kawaguchi et al., 1999), because the gain experienced by the pulses is higher at the leading edge than at the trailing edge. Figure 5(b) shows the spectral characteristics of the propagating pulse at the output of the SOA for different output energy levels. These spectral characteristics were obtained by evaluating the fast Fourier transform (FFT) of the temporal pulse shapes shown in Figure 5(a). In Fig. 5(b) we also notice that by increasing the input pulse energy, the output pulse energy increases until the SOA is driven into saturation. The dips observed at the higher frequency side of the frequency spectra are due to the self-phase modulation characteristics of the SOA (Kawaguchi et al., 1999). Also noticed that the output frequency spectra are red-shifted (the spectral peak positions are slightly shifted to the lower frequency side of the frequency spectra), and this is also attributed to the gain saturation of the SOA (Kawaguchi et al., 1999). The simulation results are in excellent agreement with the experimental results reported by Kawaguchi et al. (Kawaguchi et al., 1999). 


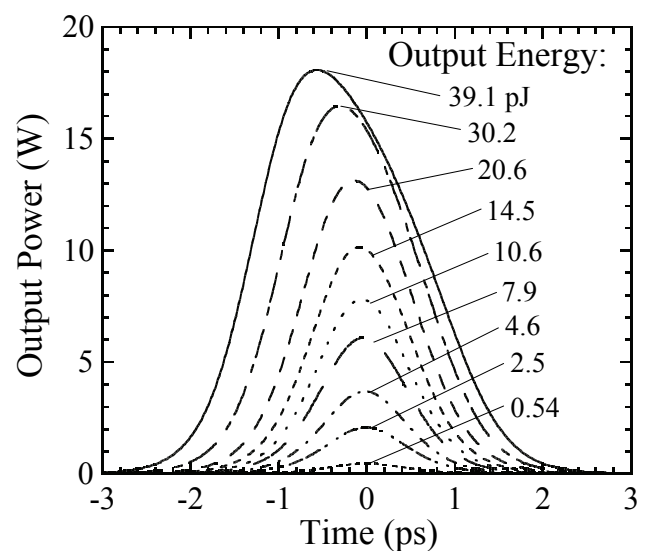

(a)

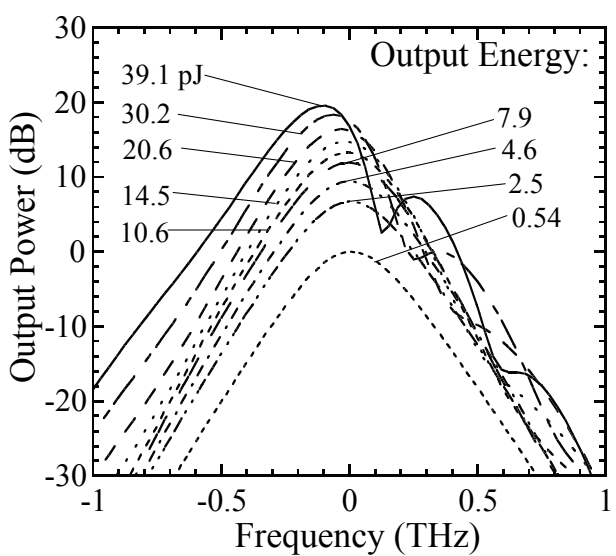

(b)

Fig. 5. (a) Temporal characteristics of the propagated pulses at the output of the SOA. Input pulse-width is 1 ps. By increasing the input pulse energy, the output energy increases until the SOA is driven into saturation. (b) Spectral characteristics of the output pulse for different output energy levels. The dips occurring at the higher frequency side are due to the self-phase modulation characteristics of the SOA.
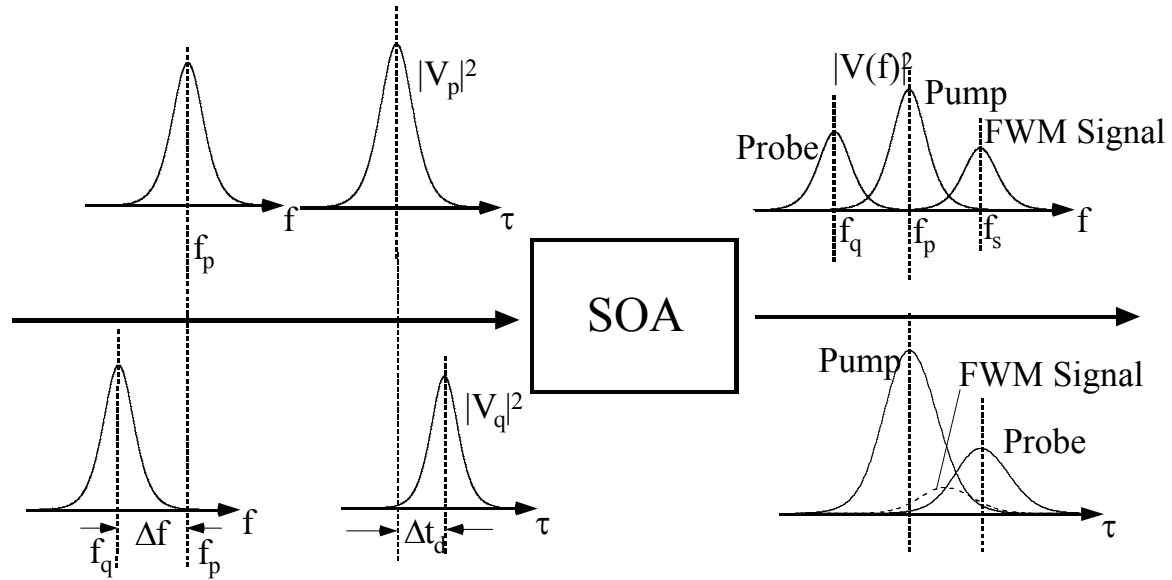

Fig. 6. Schematic diagram illustrating the input and output pump and probe pulses for the simulation of the FWM conversion efficiency in SOAs. The time delay between the input pump and probe pulses is $\Delta t_{d}$, and the detuning between the input pump and probe is $\Delta f=f_{p}-f_{q}, f_{p}$ is the center frequency of the pump pulse and $f_{q}$ is that of the probe pulse.

\subsection{FWM characteristics with time-delays between input pulses in SOAs}

When two optical pulses with different central frequencies $f_{p}$ (pump) and $f_{q}$ (probe) are injected into the SOA simultaneously, an FWM signal is generated at the output of the SOA at a frequency $2 f_{p}-f_{q}$. Figure 6 shows the schematic diagram adopted for the simulation of FWM conversion efficiency in an SOA, illustrating the time delay $\Delta t_{d}=0$ between the input 
pump and probe pulses, which are injected simultaneously into the SOA. For the analysis of the FWM conversion efficiency, the combined pump and probe pulse, $V(\tau)$, is given by

$$
V(\tau)=V_{p}(\tau)+V_{q}\left(\tau \pm \Delta t_{d}\right) \exp (-i 2 \pi \Delta f \tau)
$$

where, $V_{p}(\tau)$ and $V_{q}(\tau)$ are the complex envelope functions of the input pump and probe pulses respectively, $\tau\left(=t-z / v_{g}\right)$ is the local time that propagates with group velocity $v_{g}$ at the center frequency of an optical pulse, $\Delta f$ is detuning frequency and expressed as $\Delta f=f_{p}-f_{q}, \Delta t_{d}$ is the time-delay between the input pump and probe pulses. The positive (plus) and negative (minus) signs in $V_{q}\left(\tau \pm \Delta t_{d}\right)$ correspond to the pump leading the probe or the probe leading the pump, respectively. Using the complex envelope function of equation (16), we solved the MNLSE and obtained the distribution of the probe and pump pulses as well as the output FWM signal pulse.

For the simulations, we used the parameters of a bulk SOA (AlGaAs/GaAs, double heterostructure) at a wavelength of $0.86 \mu \mathrm{m}$. The parameters are listed in Table 1 (Hong et al., 1996). The length of the SOA was assumed to be $350 \mu \mathrm{m}$. All the results were obtained for a propagation step $\Delta \mathrm{z}$ of $5 \mu \mathrm{m}$. Note that, for any step size less than $5 \mu \mathrm{m}$ the simulation results were almost identical (i.e., independent of the step size).

\begin{tabular}{|c|c|c|c|}
\hline Name of the Parameters & Symbols & Values & Units \\
\hline Length of SOA & $\mathrm{L}$ & 350 & $\mu \mathrm{m}$ \\
\hline Effective area & $\mathrm{A}$ & 5 & $\mu \mathrm{m}^{2}$ \\
\hline Center frequency of the pulse & $\mathrm{f}_{0}$ & 349 & $\mathrm{THz}$ \\
\hline Linear gain & $\mathrm{g}_{0}$ & 92 & $\mathrm{~cm}^{-1}$ \\
\hline Group velocity dispersion & $\beta_{2}$ & 0.05 & $\mathrm{ps}^{2} \mathrm{~cm}^{-1}$ \\
\hline Saturation energy & $\mathrm{W}_{\mathrm{s}}$ & 80 & $\mathrm{pJ}$ \\
\hline Linewidth enhancement factor due to the carrier depletion & $\alpha_{N}$ & 3.1 & \\
\hline Linewidth enhancement factor due to the $\mathrm{CH}$ & $\alpha_{\mathrm{T}}$ & 2.0 & \\
\hline $\begin{array}{l}\text { The contribution of stimulated emission and FCA to the } \\
\mathrm{CH} \text { gain reduction }\end{array}$ & $\mathrm{h}_{1}$ & 0.13 & $\mathrm{~cm}^{-1} \mathrm{pJ}^{-1}$ \\
\hline The contribution of TPA & $\mathrm{h}_{2}$ & 126 & fs $\mathrm{cm}^{-1} \mathrm{pJ}^{-2}$ \\
\hline Carrier lifetime & $\tau_{\mathrm{s}}$ & 200 & ps \\
\hline $\mathrm{CH}$ relaxation time & $\tau_{\mathrm{ch}}$ & 700 & fs \\
\hline SHB relaxation time & $\tau_{\mathrm{shb}}$ & 60 & fs \\
\hline SHB saturation power & $\mathrm{P}_{\mathrm{shb}}$ & 28.3 & $\mathrm{~W}$ \\
\hline Linear loss & $\gamma$ & 11.5 & $\mathrm{~cm}^{-1}$ \\
\hline Instantaneous nonlinear Kerr effect & $\mathrm{n}_{2}$ & -0.70 & $\mathrm{~cm}^{2} \mathrm{TW}^{-1}$ \\
\hline TPA coefficient & $\gamma_{2 p}$ & 1.1 & $\mathrm{~cm}^{-1} \mathrm{~W}^{-1}$ \\
\hline $\begin{array}{l}\text { Parameters describing second-order Taylor expansion of } \\
\text { the dynamically gain spectrum }\end{array}$ & $\begin{array}{l}\mathrm{A}_{1} \\
\mathrm{~B}_{1} \\
\mathrm{~A}_{2} \\
\mathrm{~B}_{2}\end{array}$ & $\begin{array}{c}0.15 \\
-80 \\
-60 \\
0\end{array}$ & $\begin{array}{l}\text { fs } \mu \mathrm{m}^{-1} \\
\mathrm{fs} \\
\mathrm{fs}^{2} \mu \mathrm{m}^{-1} \\
\mathrm{fs}^{2}\end{array}$ \\
\hline
\end{tabular}

Table 1. Simulation parameters of a bulk SOA (AlGaAs/GaAs, double heterostructure) (Hong et al., 1996; Das et al., 2000). 


\section{Experimental setup}

Figure 7 shows the experimental setup for the measurement of the FWM signal energy at the output of the SOA with time delays being introduced between input pump and probe pulses. This experimental setup is similar to the one that was used by Inoue \& Kawaguchi (Inoue \& Kawaguchi, 1998a). In this setup, we used an optical parametric oscillator (OPO) at $1.3 \mu \mathrm{m}$ wavelength band as a light source. Here, an optical pulse train of $100 \mathrm{fs}$ was generated at a repetition rate of $80 \mathrm{MHz}$ by the OPO. The pump and probe pulses were obtained by filtering the output pulse of the OPO. The output was divided into a pump and a probe beam (pulse). Optical bandpass filters $(4 \mathrm{~nm})$ were inserted into the two beam passes to select the narrow wavelength component. After passing through the filters, the pulses were broadened to $550 \mathrm{fs}$ width, which is close to the transform-limited secant hyperbolic shape. The time-delay between pump and probe pulses is given by the optical stage (as shown in Fig. 7 time delay stage) and it regulates the optical power. The two beams were combined and injected into the SOA. The beams were amplified by the SOA and then the FWM signals were generated at the output of the SOA. The FWM signal was selected from the SOA output using two cascaded narrow-band bandpass filters ( 3 and $4 \mathrm{~nm}$ ) and detected by a photodiode. These $3 \mathrm{~nm}$ and $4 \mathrm{~nm}$ optical bandpass filters selected spectrally the FWM signal component from the output of the SOA. For the detection of FWM signal, we inserted a mechanical chopper into the probe beam path. The FWM signal was measured using the lock-in technique with $4 \mathrm{~nm}$ double-cavity bandpass filters. We adjusted the pump frequency to be $1.8 \mathrm{THz}$ higher than that of the probe frequency, i.e., the pump-probe detuning was $1.8 \mathrm{THz}$.

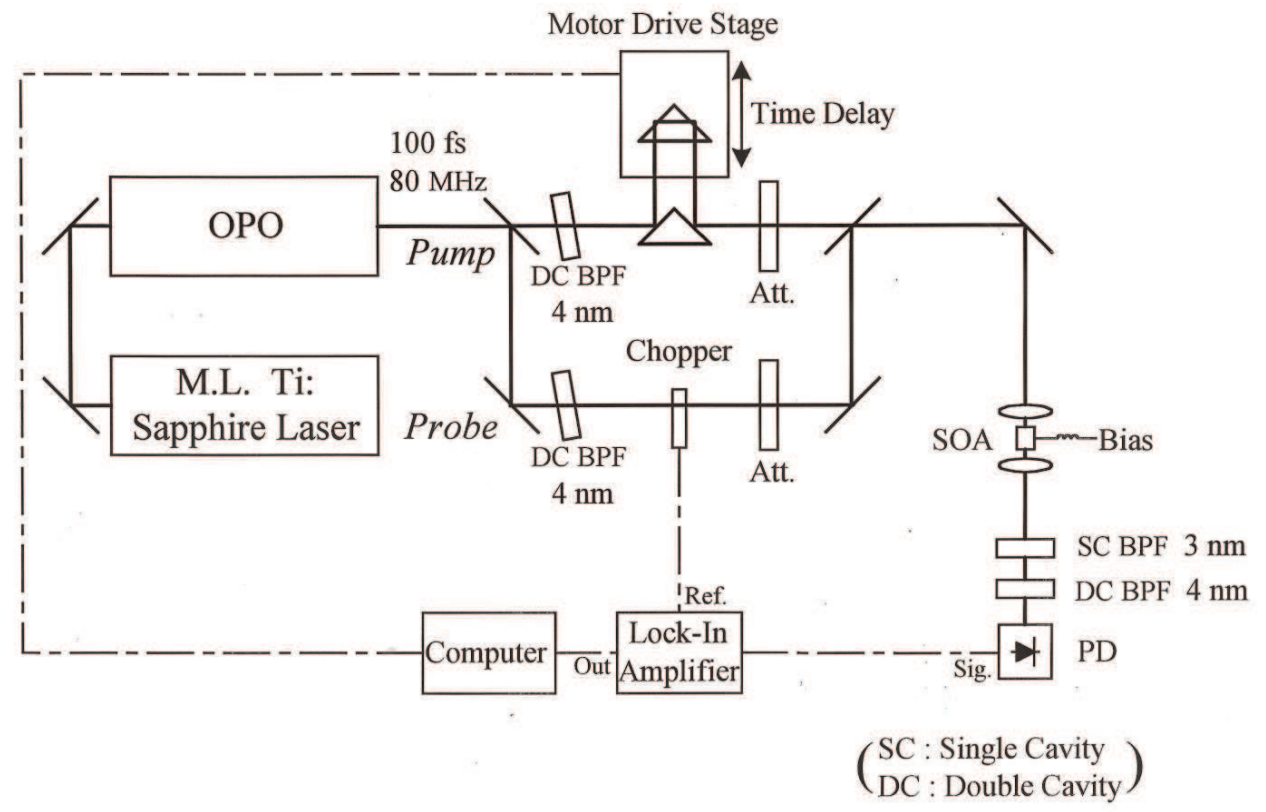

Fig. 7. Experimental setup for the measurement of FWM signal with time-delays being introduced between input pump and probe pulses in SOA. 


\section{Results and discussions}

It was found that the optimum time-delay between the input pump and probe pulses shifts from the zero time-delay $\left(\Delta t_{d}=0\right)$ under the strong input pulse condition needed to achieve high FWM conversion efficiency in an SOA. These results are very important for the design of ultrafast optical systems that have high conversion efficiency and small timing jitter (Inoue \& Kawaguchi, 1998b; Das et al., 2005).

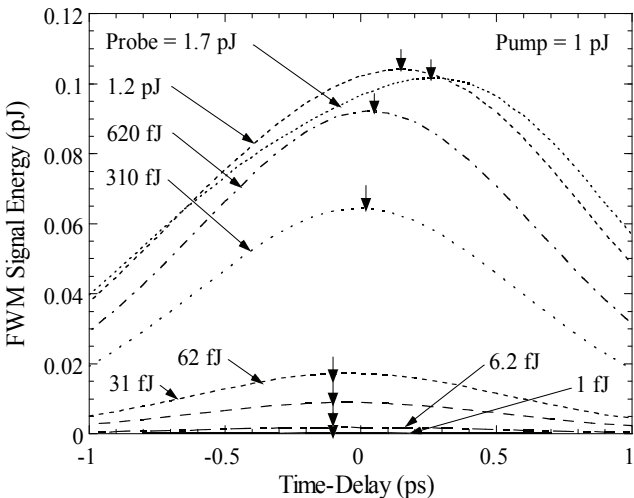

(a)

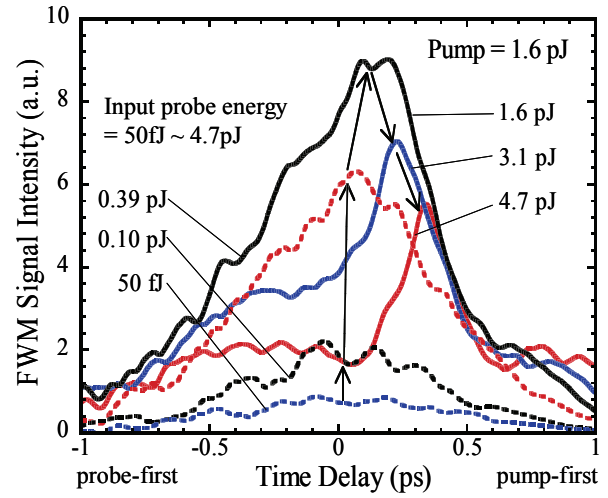

(b)

Fig. 8. (a) Simulation results: FWM signal energy (intensity) versus time-delay characteristics at the output of SOA. Input pulse-width is 1 ps and the pump-probe detuning is $3 \mathrm{THz}$. Input pump pulse energy was fixed at $1 \mathrm{pJ}$ and input probe energies were varied from $1 \mathrm{fJ}$ to $1.7 \mathrm{pJ}$. (b) Experimental results: FWM signal intensity versus timedelay. Input pulse-width is $550 \mathrm{fs}$ and pump-probe detuning is $1.8 \mathrm{THz}$. Here, the input pump pulse energy was fixed at $1.6 \mathrm{pJ}$ and input probe energies were varied from $50 \mathrm{fJ}$ to $4.7 \mathrm{pJ}$.

Figure 8(a) shows the simulation results of the FWM signal energy (intensity) at the output of the SOA versus the time-delay between the input pump and probe pulses. Here, the plus time-delay refers to the pump pulse being injected before the probe pulse. The input pump energy was fixed at $1.0 \mathrm{pJ}$. With the increase of the input probe energy, the FWM signal intensity increased until the input probe energy of $1.2 \mathrm{pJ}$, which is comparable (nearly) to the input pump energy. For a higher input probe energy $(1.7 \mathrm{pJ})$, the increase of the input probe energy decreased the FWM signal, and the peak position shifted towards the pumpfirst direction (Inoue \& Kawaguchi, 1998b; Diez et al., 1997) as illustrated by the arrows. This phenomenon is attributed to the optical nonlinear effects in the SOA, which limits the FWM conversion efficiency.

Figure 8(b) shows the experimental results of FWM signal intensity (energy) at the output of the SOA versus the time-delay between the input pump and probe pulses. For the measurements, a $1.3 \mu \mathrm{m}$ OPO system was used as a light source (experimental set up is shown in Fig. 7). The measured optimum time-delay between input pump and probe pulses 
was measured using a multiple quantum well SOA of length $350 \mu \mathrm{m}$. The shapes of the input pump and probe pulses were sech ${ }^{2}$ both had a pulse-width of $550 \mathrm{fs}$. The pump-probe detuning was set to $1.8 \mathrm{THz}$, and the input pump energy was fixed at $1.6 \mathrm{pJ}$. By increasing the input probe energy, the FWM signal intensity increased until the input probe energy became comparable to the input pump energy of $1.6 \mathrm{pJ}$. By further increasing the probe intensity, the FWM signal decreased and the peak position shifted to the pump-first direction as illustrated by the arrows. This demonstrated excellent agreement between the simulation and experimental results.

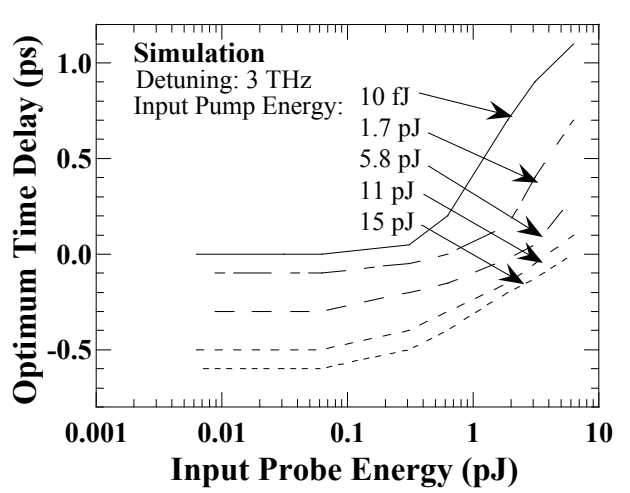

(a)

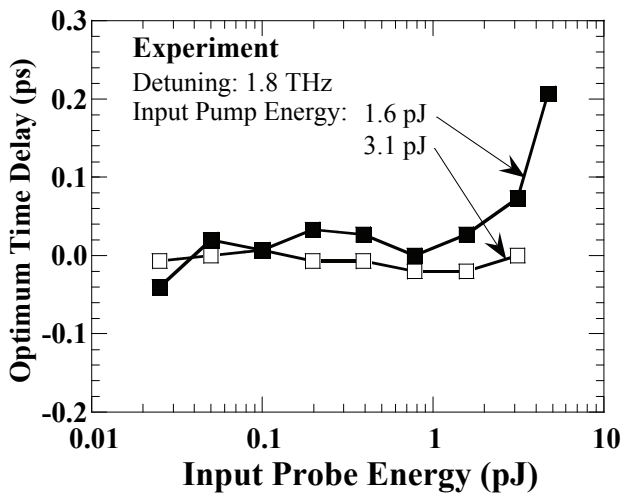

(b)

Fig. 9. (a) Simulation results: Optimum time delay versus input probe energy for different input pump energy levels. Input pulse-width $=1 \mathrm{ps}$; frequency detuning $=3 \mathrm{THz}$.

(b) Experimental results: Optimum time delay versus input probe energy for different input pump energy levels. Input pulse-width $=550 \mathrm{fs}$; frequency detuning $=1.8 \mathrm{THz}$.

Figure 9(a) shows the simulated optimum time delay between the pump and probe pulses versus the input probe energy, for an input pump and probe pulse-width of 1 ps, and a frequency detuning between pump and probe of $3 \mathrm{THz}$, and for different input pump energies. It is noticed that by increasing the input pump energy, the optimum time-delay reduces. However, increasing the input probe energy increases the optimum time delay. Figure $9(\mathrm{~b})$ shows the measured optimum time delay versus the input probe energy for different pump energy levels. The input pulse-width was $550 \mathrm{fs}$, and the frequency detuning between pump and probe was $1.8 \mathrm{THz}$. The input pump energy levels were varied between $1.6 \mathrm{pJ}$ and $3.1 \mathrm{pJ}$. Excellent agreement between the simulated and measured optimum-delay characteristics is observed for input probe energy levels above $0.1 \mathrm{pJ}$.

Figure 10(a) shows the simulated FWM conversion efficiency versus the input probe energy for different input pump energy levels. It is obvious that for a given input probe energy level, the FWM conversion efficiency increases when increasing the input pump energy level. On the other hand, for a given pump energy level, the FWM conversion efficiency decreases when the input probe energy is increased. Note that the dashed lines in Fig. 10(a) correspond to perfect pump-probe time overlap $\left(\Delta t_{d}=0\right)$, whereas solid lines correspond to optimum pump-probe time delays. Figure 10(b) shows the measured maximum FWM 
conversion efficiency (corresponding to optimum time delay between the pump and probe pulses) versus the input probe energy level for input pump energy levels $1.6 \mathrm{pJ}$ and $3.1 \mathrm{pJ}$, respectively. It is noticed from Fig. 10(b) that for a low probe energy (below 1 pJ), the FWM conversion efficiency decreases with increasing the input pump energy, whereas, for a high input probe energy (above 1 pJ), the FWM conversion efficiency increases when the input pump energy increases. From Fig. 10(a) and Fig. 10(b), excellent agreement is seen between the simulated and measured results for the optimum FWM conversion efficiency.

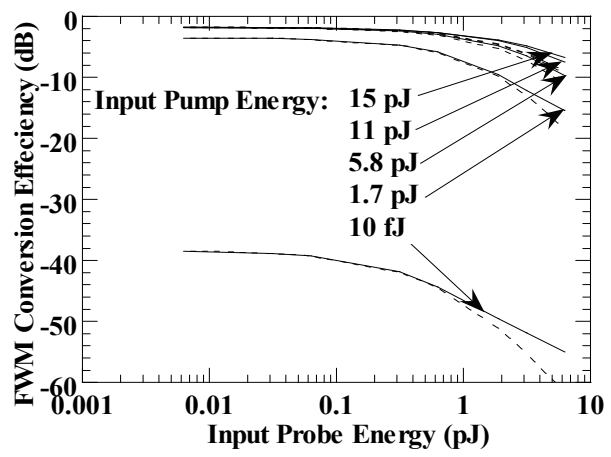

(a)

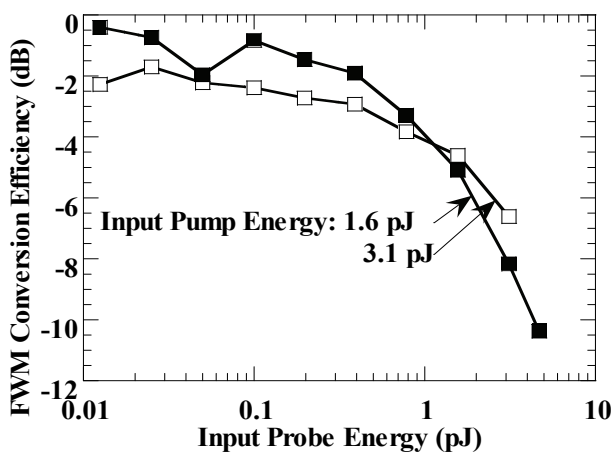

(b)

Fig. 10. (a) Simulation results: FWM conversion efficiency versus the input probe energy characteristics at the output of SOA. Dashed lines correspond to perfect pump-probe time overlap $\left(\Delta t_{d}=0\right)$, whereas solid lines correspond to optimum pump-probe time delays.

(b) Experimental results: Optimum FWM conversion efficiency versus the input probe energy characteristics for different input pump energy levels.

\section{Conclusion}

We have presented an accurate analysis based on the FD-BPM, which optimizes the time delay between the input pump and probe pulses to maximise the FWM conversion efficiency in SOAs. We have shown that the gain saturation of the SOA degrades the FWM conversion efficiency. However, by optimizing the time delay between the pump and probe pulses, for a specific pulse duration and repetition rate, a high FWM conversion efficiency can be achieved. We have also simulated and experimentally measured the optimum time delay versus the input probe energy characteristics. Simulation and experimental results have confirmed that increasing the input probe energy increases the optimum time delay and that for a low probe energy, the FWM conversion efficiency decreases with increasing the input pump energy, whereas, for a high input probe energy, the FWM conversion efficiency increases when the input pump energy is increased.

\section{Acknowledgments}

The authors would like to thank Mr. Y. Ito and Mr. Y. Yamayoshi for their helpful contribution to this work. Authors acknowledge the support of the Department of Nano-bio Materials and Electronics, Gwangju Institute of Science and Technology, Republic of Korea. 


\section{References}

Agrawal, G. P. (1989). Nonlinear Fiber Optics. Academic Press, Calif., ISBN 0-12-045142-5, San Diego.

Agrawal, G. P. \& Olsson, N. A. (1989). Self-phase modulation and spectral broadening of optical pulses in semiconductor laser and amplifiers. IEEE J. Quantum Electron., vol. 25, pp. 2297-2306, ISSN 0018-9197.

Aghajanpour, H.; Ahmadi, V. \& Razaghi, M. (2009). Ultra-short optical pulse shaping using semiconductor optical amplifier. Optics \& Laser Technology, vol. 41, pp. 654-658, ISSN 0030-3992.

Brigham, E. Oran (1988). The Fast Fourier Transform and Its Applications. Englewood Cliffs, N.J.: Prentice-Hall Inc. ISBN 0-13-307505-2.

Chung, Y. \& Dagli, N. (1990). An Assessment of finite difference beam propagation method. IEEE J. Quantum Electron., vol. 26, pp. 1335-39, ISSN 0018-9197.

Conte, S. D. \& Boor, Carl de (1980). Elementary Numerical Analysis: An Algorithmic Approach, Third Edition, McGraw-Hill Book Company Co. ISBN, Singapore. ISBN 0070124477.

Connelly, M. J.; Barry, L. P., Kennedy, B. F. \& Ried, D. A. (2008). Numerical analysis of fourwave mixing between picosecond mode-locked laser pulses in a tensile-strained bulk SOA. Optical and Quantum Electronics, vol. 40, pp. 411-418, ISSN 1572-817X.

Das, N. K.; Yamayoshi, Y. \& Kawaguchi, H. (2000). Analysis of basic four-wave mixing characteristics in a semiconductor optical amplifier by beam propagation method. IEEE J. Quantum Electron. 36, 10, pp. 1184-1192, ISSN 0018-9197.

Das, N. K. \& Karmakar, N. C. (2008). Nonlinear propagation and wave mixing characteristics of pulses in semiconductor optical amplifiers. Microwave and Optical Technology Letters, vol. 50, pp. 1223-1227, ISSN 0895-2477.

Das, N. K.; Karmakar, N. C., Yamayoshi, Y. \& Kawaguchi, H. (2007). Four-wave mixing characteristics in SOAs with optimum time-delays between pump and probe pulses," Microwave and Optical Technology Letters, vol. 49, pp. 1182-1185, ISSN 08952477.

Das, N. K.; Yamayoshi, Y., Kawazoe, T. \& Kawaguchi, H. (2001). Analysis of optical DEMUX characteristics based on four-wave mixing in semiconductor optical amplifiers. IEEE /OSA J. Lightwave Technol., vol. 19, pp. 237-246, ISSN 0733-8724.

Das, N. K.; Kawazoe, T., Yamayoshi, Y. \& Kawaguchi, H. (2001). Analysis of optical phaseconjugate characteristics of picosecond four-wave mixing signals in semiconductor optical amplifiers. IEEE J. Quantum Electron., vol. 37, pp. 55-62, ISSN 0018-9197.

Das, N. K.; Karmakar, N. C., Yamayoshi, Y. \& Kawaguchi, H. (2005). Four-wave mixing characteristics among short optical pulses in semiconductor optical amplifiers with optimum time-delays, Proceedings of the 18th Annual Meeting of the IEEE Lasers and Electro-Optics Society 2005 (IEEE-LEOS2005), pp. 127-128, ISBN 0-7803-9217-5, Sydney, NSW, Australia, October 2005, IEEE Press (USA).

Dienes, A.; Heritage, J. P., Jasti, C. \& Hong, M. Y. (1996). Femtosecond optical pulse amplification in saturated media. J. Opt. Soc. Am. B, vol. 13, pp. 725-734, ISSN 07403224. 
Diez, S.; Schmidt, C., Ludwig, R., Weber, H. G., Obermann, K., Kindt, S., Koltchanov, I. \& Petermann, K. (1997). Four-wave mixing in semiconductor optical amplifiers for frequency conversion and fast optical switching. IEEE J. Sel. Top. Quantum Electron., vol. 3, pp. 1131-1145, ISSN 1939-1404.

Inoue, J. \& Kawaguchi, H. (1998a). Highly nondegenerate four-wave mixing among subpicosecond optical pulses in a semiconductor optical amplifier," IEEE Photon. Technol. Lett., vol. 10, pp. 349-351, ISSN 1041-1135.

Inoue, J. \& Kawaguchi, H. (1998b). Time-delay characteristics of four-wave mixing among subpicosecond optical pulses in a semiconductor optical amplifier. IEEE Photon Technol. Lett. 10, pp. 1566-1568, ISSN 1041-1135.

Hong, M. Y.; Chang, Y. H., Dienes, A., Heritage, J. P., Delfyett, P. J., Dijaili, Sol \& Patterson, F. G. (1996). Femtosecond self- and cross-phase modulation in semiconductor laser amplifiers. IEEE J. Sel Top Quant Electron., vol. 2, pp. 523-539, ISSN 1939-1404.

Kawaguchi, H.; Inoue J. \& Kawazoe, T. (1999). Nonlinear propagation characteristics of subpicosecond optical pulses in semiconductor optical amplifiers. Proceedings of the 9th European Conference on Integrated Optics and Technical Exhibition, ECIO'99, pp. 455458, ISBN, Italy, April 13-16, 1999, ECIO, Triono.

Leuthold, J.; Mayer, M., Eckner, J., Guekos, G., Melchior, H. and Zellweger, Ch. (2000). Material gain of bulk $1.55 \mu \mathrm{m}$ InGaAsP/InP semiconductor optical amplifiers approximated by a polynomial model. J. Appl. Phys., vol. 87, pp. 618-620, ISSN.

Mecozzi, A.; D’Ottavi, A., Iannone, E., \& Spano, P. (1995). Four-wave mixing in travellingwave semiconductor amplifiers. IEEE J. Quantum Electron., vol. 31, pp. 689-699, ISSN 0018-9197.

Mecozzi, A. \& Mфrk, J. (1997). Saturation effects in nondegenerate four-wave mixing between short optical pulses in semiconductor laser amplifiers. IEEE J. Sel Top Quantum Electron., vol. 3, pp. 1190-1207, ISSN 1939-1404.

Okamoto, K. (1992). Theory of Optical Waveguides, Corona Publishing Co., Tokyo; Ch. 7 (in Japanese). ISBN 4-339-00602-5.

Razaghi, M.; Ahmadi, A., Connelly, M. J. \& Madanifar, K. A. (2009a). Numerical modelling of sub-picosecond counter propagating pulses in semiconductor optical amplifiers, Proceedings of the 9th International Conference on Numerical Simulation of Optoelectronic Devices 2009 (NUSOD' 09), pp. 59-60, ISBN 978-1-4244-4180-8, GIST, Gwangju, South Korea, September 2009, IEEE Press (USA).

Razaghi, M.; Ahmadi, A., \& Connelly, M. J. (2009b). Comprehensive finite-difference timedependent beam propagation model of counter propagating picosecond pulses in a semiconductor optical amplifier. IEEE /OSA J. Lightwave Technol., vol. 27, pp. 31623174, ISSN 0733-8724.

Sauter, E. G. (1996). Nonlinear Optics. John Wiley \& Sons, Inc. New York. ISBN 0-47114860-1.

Seki, K.; Kamiya, T. and Yanai, H. (1981). Effect of waveguiding properties on the axial mode competition in stripe-geometry semiconductor lasers. IEEE J. Quantum Electron., vol. 17, pp. 706-713, ISSN 0018-9197. 
Shtaif, M. \& Eisenstein, G. (1995). Analytical solution of wave mixing between short optical pulses in semiconductor optical amplifier. Appl Phys. Lett. 66, pp. 1458-1460, ISSN 0003-6951.

Shtaif, M.; Nagar, R. \& Eisenstein, G. (1995). Four-wave mixing among short optical pulses in semiconductor optical amplifiers. IEEE Photon Technol. Lett. 7, pp. 1001-1003. ISSN 1041-1135.

Yariv, A. (1991). Optical Electronics, 4th Edition, Saunders College Publishing, San Diego. ISBN 0-03-053239-6. 


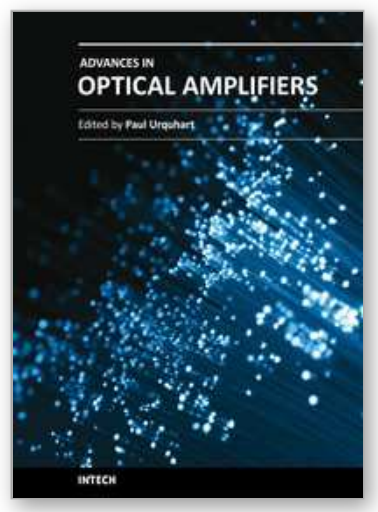

\author{
Advances in Optical Amplifiers \\ Edited by Prof. Paul Urquhart
}

ISBN 978-953-307-186-2

Hard cover, 436 pages

Publisher InTech

Published online 14, February, 2011

Published in print edition February, 2011

Optical amplifiers play a central role in all categories of fibre communications systems and networks. By compensating for the losses exerted by the transmission medium and the components through which the signals pass, they reduce the need for expensive and slow optical-electrical-optical conversion. The photonic gain media, which are normally based on glass- or semiconductor-based waveguides, can amplify many high speed wavelength division multiplexed channels simultaneously. Recent research has also concentrated on wavelength conversion, switching, demultiplexing in the time domain and other enhanced functions. Advances in Optical Amplifiers presents up to date results on amplifier performance, along with explanations of their relevance, from leading researchers in the field. Its chapters cover amplifiers based on rare earth doped fibres and waveguides, stimulated Raman scattering, nonlinear parametric processes and semiconductor media. Wavelength conversion and other enhanced signal processing functions are also considered in depth. This book is targeted at research, development and design engineers from teams in manufacturing industry, academia and telecommunications service operators.

\title{
How to reference
}

In order to correctly reference this scholarly work, feel free to copy and paste the following:

Narottam Das, Hitoshi Kawaguchi and Kamal Alameh (2011). Impact of Pump-Probe Time Delay on the Four Wave Mixing Conversion Efficiency in Semiconductor Optical Amplifiers, Advances in Optical Amplifiers, Prof. Paul Urquhart (Ed.), ISBN: 978-953-307-186-2, InTech, Available from:

http://www.intechopen.com/books/advances-in-optical-amplifiers/impact-of-pump-probe-time-delay-on-thefour-wave-mixing-conversion-efficiency-in-semiconductor-optic

\section{INTECH}

open science / open minds

\section{InTech Europe}

University Campus STeP Ri

Slavka Krautzeka 83/A

51000 Rijeka, Croatia

Phone: +385 (51) 770447

Fax: +385 (51) 686166

www.intechopen.com

\section{InTech China}

Unit 405, Office Block, Hotel Equatorial Shanghai

No.65, Yan An Road (West), Shanghai, 200040, China

中国上海市延安西路 65 号上海国际贵都大饭店办公楼 405 单元

Phone: +86-21-62489820

Fax: $+86-21-62489821$ 
(C) 2011 The Author(s). Licensee IntechOpen. This chapter is distributed under the terms of the Creative Commons Attribution-NonCommercialShareAlike-3.0 License, which permits use, distribution and reproduction for non-commercial purposes, provided the original is properly cited and derivative works building on this content are distributed under the same license. 